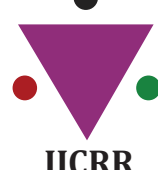

IJCRR

Section: Healthcare Sci. Journal Impact Factor: 5.385 (2017) ICV: 71.54 (2015)

\title{
Efficacy of Four Square Step Test and Modified Dynamic Gait Index to Predict the Falls in Parkinson's Disease: A Comparative Study
}

\author{
Snehal Joshi ${ }^{1}$, Swapnali Joshi ${ }^{2}$
}

'Professor, D.E. Society's Brijlal Jindal College of Physiotherapy, Fergusson College Campus, Shivajinagar, Pune-4110o5; ${ }^{2}$ M.P.Th., D.E. Society's Brijlal Jindal College of Physiotherapy, Fergusson College Campus, Shivajinagar, Pune-411005.

\section{ABSTRACT}

Introduction: This study evaluated the efficacy of Four square step test (FSST) and modified dynamic gait index (mDGI) in patients with Parkinson's disease to evaluate balance. In this study 50 individuals with Parkinson's disease were assessed according to Hoen Yahr scale (grades between 1to 3), were subjected to FSST and mDGI.

Results: FSST and m DGI scores were taken of the same patient. and sensitivity and specificity were also calculated. Both these scales show a highly significant negative correlation with each other $(. r=-0.88, P<0.001)$

Conclusion: Thus the study concluded that both FSST and MDGI are equally effective for evaluating balance in individuals with Parkinson's disease.

Key Words: Sensitivity, Specificity, Balance, Parkinson's disease

\section{INTRODUCTION}

Parkinson's disease (PD) is a progressive disorder characterized by various motor and non-motor features that can impact on function a variable degree. The four cardinal features of PD that can be under the acronym TRAP: Tremor at rest, rigidity, Akinesia (or bradykinesia) and Postural inability. In addition, flexed posture and freezing (motor blocks) are classic features of parkinsonism, with PD as the most common form. ${ }^{1}$

The symptoms of PD begin insidiously and worsen gradually. Rest tremor is often the first symptom recognized by the patient. But sometimes it begins with bradykinesia; and in some patients, tremor may never develop. Bradykinesia manifests as slowness, such as slower and smaller handwriting, decreased arm swing and leg stride when walking, decreased facial expression, and decreased amplitude of voice.
Rest tremor can be intermittent at the beginning, might be present only in stressful situations; eventually it tends to be present most of the time and got worsens in amplitude with stress or excitement. ${ }^{2}$ There is a continuous worsening of symptoms over time; and if it remains untreated then those symptoms may lead to disability with severe immobility and falling tendency may increase. The early symptoms and signs of PD that is rest tremor, bradykinesia, and rigidity are related to progressive loss of nigrostriatal dopamine. ${ }^{2}$ These signs and symptoms result from striatal dopamine deficiency and are usually correctable by levodopa and dopamine agonists. As PD progresses over time, symptoms that do not respond to levodopa develop, such as flexed posture, the freezing phenomenon, and loss of postural reflexes; these are often referred to as non-dopamine-related features of PD. Moreover, bradykinesia that responded to levodopa in the early stage of PD increases as the disease worsens

Corresponding Author:

Snehal Joshi, Professor, D.E. Society's Brijlal Jindal College of Physiotherapy, Fergusson College Campus, Shivajinagar, Pune-411005. Contact no.: +91-9822490291; Email: drsnehalmandke@gmail.com

ISSN: 2231-2196 (Print)

Received: 24.06 .2019
ISSN: 0975-5241 (Online)

Revised: 12.08 .2019
Accepted: 12.09 .2019 
and no longer fully responds to levodopa. It is particularly these intractable motoric symptoms that lead to the disabilities of increasing immobility and balance difficulties. There are also some non-motor symptoms associated with motor symptoms, these include fatigue, depression, anxiety, sleep disturbances, constipation, bladder and other autonomic disturbances (sexual, gastrointestinal), and sensory complaints. Sensory symptoms include pain, numbness, tingling, and burning in the affected limbs; Behavioral and mental alterations are common and include changes in mood, decreased motivation and apathy, slowness in thinking (bradyphrenia), and a declining cognition that can progress to dementia. ${ }^{2}$

\section{Stages of Parkinson disease according to Hoen Yahr scale ${ }^{3}$}

Hoen and Yahr Scale

1: Only unilateral involvement, usually with minimal or no functional disability

2: Bilateral or midline involvement without impairment of balance

3: Bilateral disease: mild to moderate disability with impaired postural reflexes; physically independent

4: Severely disabling disease; still able to walk or stand unassisted

5: Confinement to bed or wheelchair unless aided

\section{Motor symptoms of Parkinson's include:}

Tremor, bradykinesia, rigidity, postural instability, Hypomimia, dysarthria, dysphagia, sialorrhoea, decreased arm swing, shuffling gait, loss of righting reactions, festination difficulty arising from chair, turning in bed, Micrographia, slow activities of daily living, Glabellar reflex, blepharospasm, dystonia, scoliosis, camptocormia

Balance impairment or postural instability is one of the major problems faced by Parkinson's patients. This can lead to falls which in turn can lead to serious consequences like death or morbidity. Hence it IS necessary to use an outcome measure which will determine the individuals at the risk of fall

- A number of rating scales are used for the evaluation of motor impairment and disability in patients with PD, like UPDRS', modified dynamic gait index ( $\mathrm{m}$ DGI), four square step test ( FSST), timed up and go test (TUG), etc. ${ }^{1}$

Balance is one of the important parts of day to day life. Gait and balance deficits are common in patients with Parkinson disease. Patients with PD are characterized clinically by movement-related symptoms, such as tremor, rigidity, slow movement (bradykinesia), and postural instability.
Consequently, they experience difficulties in gait and gait related activities (e.g., turning and climbing stairs) that reduce their quality of life.

To manage and monitor gait and balance deficits, clinicians need to routinely measure these characteristics of patients with PD. Furthermore, to interpret the results of the measurements, clinicians must determine whether change scores in gait and balance deficits represent true changes or are a result of measurement error.

There are so many outcome measures which are used for assessing balance in individuals with Parkinson's disease like dynamic gait index, berg balance scale, timed up and go test, four square step test, etc. Amongst them modified dynamic gait index is used as gold standard outcome measure to assess balance in Parkinson's disease individuals as its reliability and validity is very high in Parkinson's disease.

Recent studies found that four square step test is also valid in individuals with Parkinson's disease. Four square step test is short, less time consuming, easy test to assess balance. In Parkinson's disease individuals usually fatigue easily due to posture, age, severity of disease, bradykinesia. Other balance tests are quiet time consuming $\mathrm{n}$ lengthy which causes individuals to fatigue in test, so that it is not possible to get effective outcome of those test.

There are very few studies are conducted on four square step test in Parkinson's disease individuals. There are no studies are performed on comparison of four square step test and modified dynamic gait index in individuals with Parkinson's disease individuals.

So purpose of this study was to find out correlation between four square step test and modified dynamic gait index and its sensitivity and specificity in individuals with Parkinson's disease.

\section{Four square step test ${ }^{4}$ (FSST):}

The Four Square Step Test (4SST), first described by Dite and Temple, is a quick and simple clinical test that requires an individual to rapidly step over obstacles in the forward, backward, and sideways directions. It was hypothesized that the 4SST may be a useful balance screen in people with PD for the following reasons: a) it demands complex anticipatory postural adjustments for gait initiation, known to be impaired in people with $\mathrm{PD}, \mathrm{b}$ ) the requirements for stepping backward and laterally may be particularly challenging for people with $\mathrm{PD}$, and c) the task requires execution of a complex multi-step movement sequence, another area of known difficulty in PD. ${ }^{4}$

Four square step test versus Modified Dynamic Gait Index in Parkinson's disease 


\section{Modified dynamic gait index (mDGI):}

A modified version of the DGI (mDGI) was recently developed by Shumway-Cook and colleagues. The mDGI retains the original 8 tasks but expands the scoring system to evaluate three correlated but unique aspects of walking performance like gait pattern, level of assistance, and time. ${ }^{7}$

The original 8 tasks of DGI were retained in the mDGI; however, minor modifications were made to facilitate timing and to clarify procedures for several of the tasks. To enable timing, a 6.1-m distance was imposed for all tasks. The change pace task included an acceleration phase ("walk as quickly as you safely can") but no deceleration phase, as it was difficult to impose both of these task modifications in a distance of $6.1 \mathrm{~m}$. The over obstacle task changed the dimensions of the obstacles and specified the dimensions and placement of the 2 obstacles.

\section{AIM}

To compare efficacy of four square step test and modified dynamic gait index in individuals with Parkinson's disease.

\section{OBJECTIVES}

1. To find out correlation between four square step test and modified dynamic gait index

2. To find out sensitivity and specificity of four square step test in Parkinson's patient.

3. To compare sensitivity and specificity of modified dynamic gait index in Parkinson's patient.

\section{MATERIALS AND METHODOLOGY}

Type of Study: Observational analytical cross sectional study

Study Population: Individuals with Parkinson's disease

\section{Inclusion Criteria:}

1. Individuals with parkinsonism disease

2. Individuals who are in between Hoen Yahr grade 1 to 3.

\section{Exclusion Criteria:}

1. Affection in cognition and other perceptual disorders

2. Visual problem

3. Patients having dementia

4. Auditory problems

\section{MATERIALS}

Outcome measures: Four square step test (FSST), Modified dynamic gait index (mDGI).

1. Ribbons

2. Measuring tape

3. Stop watch

4. $76 \mathrm{~cm}$ long, $12 \mathrm{~cm}$ wide, $5 \mathrm{~cm}$ thick obstacle

Study settings: Physiotherapy OPD, Patient's home

Sampling technique: Simple random sampling

Sample size: 50 subjects

\section{METHODOLOGY}

The clearance from the ethics committee was taken.

The subjects were screened according to the inclusion, exclusion criteria.

An informed written consent was taken from the subject. The entire procedure was explained to the subject. Before the administration of balance outcome measures, Hoen Yahr scale and history of fall was assessed. Then subjects were asked to perform Four square step test. FSST was assessed twice and best time taken as the score. ${ }^{4}$ After taking 10 minutes of rest subjects were asked to perform modified dynamic gait index. Data was analyzed using spearman correlation test for correlation. The sensitivity and specificity will be calculated using formula:

\section{RESULTS}

Fifty subjects were included in this study after screening for inclusion and exclusion criteria.

For finding cutoff point of Modified dynamic gait index\& FSST ROC curve was plotted

Statistical analysis for correlation of Four square step test and Modified dynamic gait index was done using Spearman's correlation formula.

Sensitivity and specificity of Four square step test and Modified dynamic gait index was done using sensitivity and specificity formula.

\section{Table I: Age Distribution}

\begin{tabular}{llllll} 
& $\mathrm{N}$ & Minimum & Maximum & Mean & Std. Deviation \\
$\begin{array}{l}\text { Age in } \\
\text { years }\end{array}$ & 50 & 82 & 65.5 & +_9.181 \\
\hline
\end{tabular}




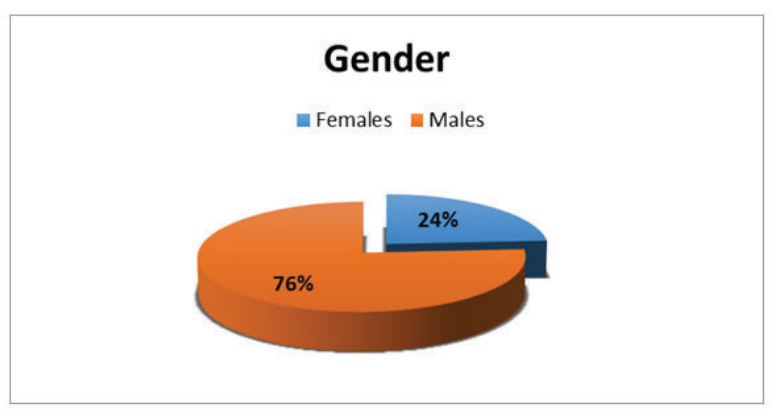

Graph I: Gender Distribution.

Graph I indicate gender distribution in subjects. It showed that around $76 \%$ were males and $24 \%$ were female

\section{Table II: HOEN YAHR Scale Distribution}

\begin{tabular}{lc} 
HYS (Grades) & No. of subjects \\
1 & 19 \\
2 & 21 \\
3 & 10 \\
Total & 50 \\
\hline
\end{tabular}

Table III: Sensitivity and Specificity of FSST and mDGI

\begin{tabular}{lccc} 
mDGI & \multicolumn{2}{c}{ FSST } & Total \\
& $>9.68$ & $\leq 9.68$ & \\
$\leq 56$ & 31 & 3 & 34 \\
$>56$ & 3 & 13 & 16 \\
Total & 34 & 16 & 50 \\
\hline
\end{tabular}

Table III indicates sensitivity and specificity of mDGI and FSST.

mDGI Sensitivity $=91.2 \%$ Specificity $=81.2 \%$

FSST Sensitivity $=94 \%$ Specificity $=84 \%$

For finding cutoff point of Modified dynamic gait index ROC curve was plotted.

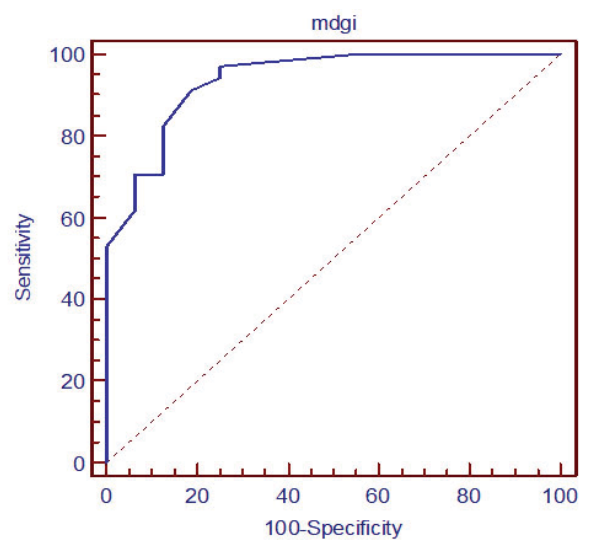

Graph II: ROC curve of mDGI.
Graph II shows cutoff point for mDGI is 56 . At this cutoff, maximum sensitivity and specificity can be established.

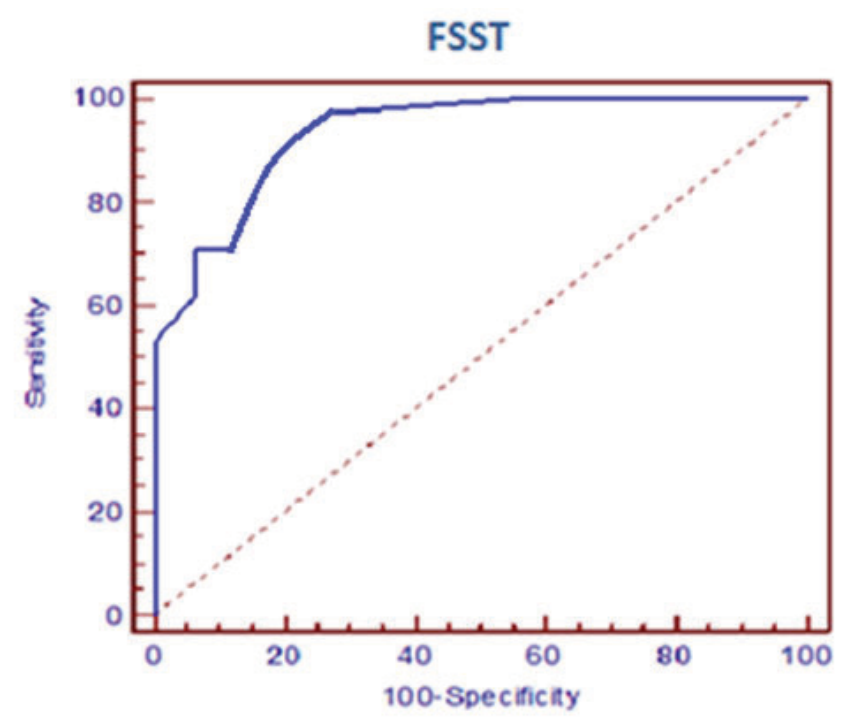

Graph III: ROC curve of FSST.

GRAPH IV shows cutoff point for FSST is 9.68 seconds.

At this cutoff maximum sensitivity and specificity can be established

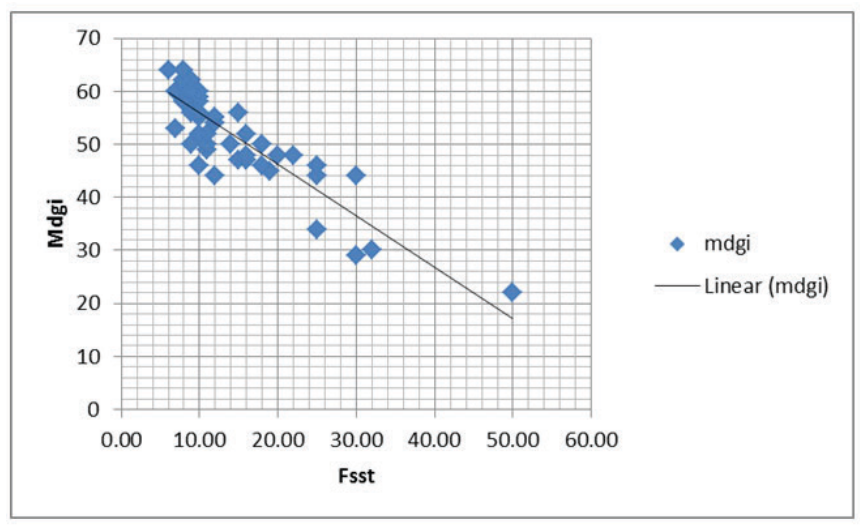

Graph IV: Correlation coefficient of FSST and MDGI.

Graph IV indicates highly significant negative correlation between FSST and mDGI. $(\mathrm{r}=-0.88, \mathrm{P}<0.001)$

\section{DISCUSSION}

The main focus of the study was to compare efficacy of four square step test and modified dynamic gait index in individuals with Parkinson's disease.

Total 50 subjects were assessed for this study. Each subject was asked to perform four square step test and modified dynamic gait index. 
The loss of DA from the substantia nigra leads to alterations in both the direct and indirect pathways of the basal ganglia, resulting in the decrease in excitatory thalamic input to the cortex and perhaps a decrease in inhibitory surround that leads to the symptoms of Parkinson's disease ${ }^{5}$.

As might be suspected from the review of functional physiology of the basal ganglia, the postural abnormalities include an assumption of a flexed posture, lack of equilibrium reactions, specially of the labyrinthine equilibrium reactions and a decrease in trunk rotation ${ }^{6}$.

Individuals with Parkinson's disease also demonstrate hypokinesia, defined as movements that are decreased in amplitude and Akinesia, or the reduced ability to initiate movement ${ }^{7}$.

Postural instability is a serious problem in Parkinsonism that leads to increased episodes of falling and the sequel of falls $s^{1,8}$. Patients have increased likelihood of falling as the duration of the disease increases. Drug treatment is not usually effective in reducing the incident of falls.

Bradykinesia is one of important features of PD. Bradykinesia is defined as slowness of movement, that means time taken to execute a task specific movement once it has been initiated. ${ }^{1,9,10,7}$

Bradykinesia could potentially be due to slowness in formulating instructions to programming or to slowness in executing theses instructions. ${ }^{6}$ Movements are typically reduced in speed, range and amplitude. Rigidity and depression can also influence bradykinesia. Often it is the most disabling symptom of PD, with the slowness and prolonged movement times leading to increased dependence in daily task. ${ }^{6,7}$

In FSST time required to complete the test is measured. As in individuals with Parkinson's disease have bradykinesia and impaired reaction time as well as equilibrium reaction. This test might have shown high sensitivity and specificity in individuals with Parkinson disease ${ }^{6}$.

In mDGI, all components are assessed according to time, level of assistance and gait pattern. In Parkinson's disease due to basal ganglia affection motor symptoms like Akinesia, bradykinesia, rigidity, tremors etc. also slowness in equilibrium reactions are seen that may cause increase in time and loss of balance to complete the task. Also Parkinson patient may need assistive devices due to these motor symptoms. Due to these motor affection Parkinson patient find difficulty in gait. So all these components are assessed in mDGI.

As mDGI assesses reaction time, bradykinesia and equilibrium reaction with respect to time, level of assistance and gait, so it might have shown good sensitivity and specificity in individuals with Parkinson disease.

As both the tests, FSST and mDGI, assess same components of balance that is bradykinesia, Akinesia and equilibrium re- actions in time limit, they have shown a highly significant negative correlation.

That means as the score on the mDGI increases the time taken to complete FSST is decreases which means less affected balance in individual with Parkinson disease.

As mDGI has 8 components for assessment also it gives detailed evaluation according to time, level of assistance and gait pattern, it needs larger space but takes more time.

Four square step test can be used as an equally effective assessment also it needs small space and less time to perform the test

So mDGI can be used for more of research purpose whereas FSST can be used as clinical assessment tool.

\section{CONCLUSION}

This study concluded that FSST \& mDGI are equally effective for assessing balance in Parkinson's patients.

As mDGI has 8 components for assessment also it gives detailed evaluation according to time, level of assistance and gait pattern and thus can be more helpful in research whereas Four square step test can be used as effective assessment tool in routine clinical set up as it needs small space and less time to perform the test.

Studies can be conducted to determine efficacy of different interventions using these two scales as outcome measures.

Funding: No funding available

Conflict of interest: No conflict of Interest

\section{ACKNOWLEDGMENT}

Authors acknowledge the immense help received from the scholars whose articles are cited and included in references of this manuscript. The authors are also grateful to authors / editors / publishers of all those articles, journals and books from where the literature for this article has been reviewed and discussed

\section{REFERENCES}

1. J Jankovic Parkinson's disease: clinical features and diagnosis J Neurol Neurosurg Psychiatry 2008; 79:368-376. doi:10.1136/ jnnp.2007.131045.

2. Stanley Fahn Description of Parkinson's Disease as a Clinical Syndrome Ann. N.Y. Acad. Sci. 991: 1-14 (2003). (C) 2003 New York Academy of Sciences.

3. R. Bhidayasiri, D. Tarsy, Movement Disorders: A Video Atlas"Current Clinical Neurology, DOI 10.1007/978-1-60327426-5_2, (C) Springer Science Business Media New York 2012 
4. Adriana Galvan et al Pathophysiology of Parkinsonism Clinical Neurophysiology. 2008 July; 119(7): 1459-1474.

5. P. Santens, et al The pathophysiology of motor symptoms in Parkinson's disease Acta neurol. belg., 2003,103, 129-134.

6. Allan H. Ropper, Martin A. Samuels, Joshua P. Klein Adams and Victor's Principles of Neurology $10^{\text {th }}$ edition Ch.4 Abnormalities of Movement and Posture Caused by Disease of the Basal Ganglia.

7. Boonstra, Tjitske et al Gait disorders and balance disturbances in Parkinson's disease: clinical update and pathophysiology Move- ment disorders: Edited by Marie Vidailhet August 2008 - Volume 21 - Issue 4 - p 461-471.

8. B H Wood, et al Incidence and prediction of falls in Parkinson's disease: a prospective multidisciplinary study J Neurol Neurosurg Psychiatry 2002;72:721-725.

9. Isao Sugeta, Rpt, Ms et al Ability of the Four-square Step Test to Predict Falls among Community-dwelling Frail Elderly Rigakuryoho Kagaku 2016 31(4): 615-620,

10. Shumway-Cook et al Modified Dynamic Gait Index Physical Therapy Volume 93(11) 2013 ; 1493-1506. 